\title{
Comments On Slawinski's Paper
}

Keith Harary, Ph.D.

Institute for Advanced Psychology

Janusz Slawinski has some interesting ideas about life after death, which would be thought provoking if I believed that consciousness has an electromagnetic nature. But there are a number of reasons why I'm not convinced that that is the case.

First, experiments in psi research have demonstrated that the accuracy and reliability of psychic abilities like remote viewing don't fall off as a function of distance, electromagnetic shielding, or time. Precognition appears to work as well as real-time remote viewing. Viewers in a submerged submarine were able to describe accurately the activities of target persons on land, despite the electromagnetic blocking effects of sea water. Remote viewing across thousands of miles appears to be no less accurate than viewing into the next room. In short, this intriguing conscious process doesn't appear to function according to known laws of electromagnetic interaction.

Second, we saw no consistent evidence in our out-of-body experience (OBE) research at Duke University to suggest that any electromagnetic radiation is associated in an obvious way with OBEs. Of course, we focused primarily upon measuring possible electromagnetic radiation effects or light emissions in the "detection area," and not in the "launch area" immediately around the body itself. There were simply not enough resources to carry out every conceivable experiment.

Personally, I am not convinced that consciousness is ever actually confined to a body to begin with. My experience as a psi researcher, and my own personal experience, suggest to me that consciousness is related to some deep interconnectedness in nature that appears to be

Dr. Harary is President and Research Director of the Institute for Advanced Psychology. Requests for reprints should be sent to Dr. Harary at the Institute for Advanced Psychology, 1550 California Street, San Francisco, CA 94109. 
quite independent of human observers. The image of life being composed of individual "packets" of consciousness, memories, ego, or whatever seems to be more a product of our limited perceptions of reality than of actual underlying processes in nature. Before we consider whether our "packets" survive death, we need to define what we think we mean by life. 